NASA Technical Memorandum 84663

\title{
A Finite Difference Solution for the Propagation of Sound in Near Sonic Flows
}

S. I. Hariharan and Harold C. Lester

FOR PEFERENCE

JULY 1983

Lenher mory

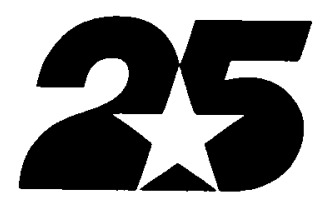

25th Anniversary

1958-1983

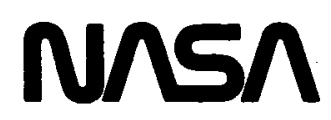



NASA Technical Memorandum 84663

\section{A Finite Difference Solution for the Propagation of Sound in Near Sonic Flows}

S. I. Hariharan

Institute for Computer Applications in Science and Engineering Langley Research Center

Hampton, Virginia

Harold C. Lester

Langley Research Center

Hampton, Virginia

\section{N/SA}

National Aeronautics

and Space Administration

Scientific and Technical

Information Branch

1983 

SUMMARY

An explicit time/space finite difference procedure is used to model the propagation of sound in a quasi one-dimensional duct containing high Mach number subsonic flow. Nonlinear acoustic equations are derived by perturbing the time-dependent Euler equations about a steady, compressible mean flow. The governing difference relations are based on a fourth-order, two-step (predictor-corrector) MacCormack scheme. Difference equations for the source and termination boundary conditions are derived from the appropriate characteristic relationships. The solution algorithm functions by switching on a time harmonic source and allowing the difference equations to iterate to a steady state. A significant advantage with this approach is that the nonlinear terms can be retained and evaluated with only modest additional computer cost above that required for a linear model calculation.

The principal effect of the nonlinearities was to shift acoustical energy to higher harmonics. With increased source strengths, wave steepening was observed. This phenomenon suggests that the acoustical response may approach a shock behavior at a higher sound pressure level as the throat Mach number approaches unity. Where applicable, comparisons were made with the calculations from a linear finite element algorithm. On a peak level basis, good agreement between the nonlinear finite difference and linear finite element solutions was observed, even though a peak sound pressure level of about $150 \mathrm{~dB}$ occurred in the throat region. Nonlinear steady state waveform solutions are shown to be in excellent agreement with a nonlinear asymptotic theory.

\section{INTRODUCTION}

It has been customary in computational duct acoustics to base calculative procedures on steady state linear equations using direct implicit numerical schemes. The Galerkin/weighted-residual method summarized in reference 1 is a state-of-the-art example of this approach. Such direct methods, whether based on finite element or finite difference methodology (ref, 2), produce a large set of coupled linear equations, which are usually solved by matrix manipulations, that is, inversion/ decomposition procedures. However, many practical aeroengine duct modeling situations require extremely large numbers of degrees-of-freedom for accurate resolution. Not only are the direct methods costly in computer time for these problems, but in many instances, the computer core storage limitations are exceeded, and expensive out-of-core solution algorithms are necessary.

Recently, Baumeister (refs. 3 to 5) advocated the use of explicit time-dependent difference methods for steady state duct acoustic calculations. With these methods, the time-dependent equations, subject to a harmonically varying source boundary condition, are iterated over a time/space difference discretization grid until steady state is achieved. Such procedures have the advantage of tremendously reducing core storage requirements. In addition, both linear and nonlinear equations can be handled, since the nonlinear terms are simply retained in the difference relations and evaluated during each iterative calculation step with a minimum of additional cost. 
In this paper, an explicit time/space finite difference procedure is used to simulate the propagation of sound in a quasi one-dimensional variable area duct containing high Mach number subsonic flow. For this physical situation it has been shown, both analytically and experimentally (refs. 6 to 8, for example), that significant intensification of the acoustic wave structure occurs in the throat region as the flow velocity approaches sonic value. In fact, linear theory is singular for a throat Mach number of unity (ref. 6) and is therefore invalid in the throat region. Nonlinear acoustic equations are derived by perturbing the time-dependent Euler equations about a steady compressible state. The acoustic equations are then cast into a nondimensional form and discretized in space and time by a fourth-order, two-step (predictor-corrector) MacCormack difference scheme (ref. 9). Numerical boundary conditions are derived for the source and termination sections from the appropriate characteristic relationships. The solution algorithm functions by switching on a real time harmonic source and allowing the difference equations to iterate to a steady state condition.

Calculated results are presented for several area reduction ratios, throat Mach numbers, and acoustic source sound pressure levels. Where applicable, comparisons are made with the results calculated by a linear Galerkin/weighted-residual finite element algorithm. At higher sound pressure levels and throat Mach numbers approaching unity, for which nonlinear effects become significant, results calculated by the finite difference procedure are compared with the asymptotic theory developed by Callegari and Myers (ref, 10).

SYMBOLS

A

c

$\mathbf{f}$

$\mathrm{J}$

j

k

L

M

N

n

p

$S$

$\mathrm{T}_{1}, \mathrm{~T}_{2} \quad$ characteristic quantities, equations (19) and (21)

$t \quad$ time

area variation

speed of sound

frequency

number of grid points

node index, $x=(j-1) \Delta x$

wave number, $k=\omega / c_{0}$

length of flow duct

Mach number

number of time steps

time index, $t=n \Delta t$

acoustic pressure

source amplitude 


$\begin{array}{ll}\mathbf{u} & \text { acoustic particle velocity } \\ \overline{\mathbf{u}} & \text { acoustic streaming velocity } \\ \mathbf{x} & \text { axial coordinate } \\ \gamma & \text { ratio of specific heats } \\ \Delta \mathrm{x} & =\mathrm{L} /(\mathrm{J}-1) \\ \varepsilon & \text { perturbation factor } \\ \rho & \text { acoustic density } \\ \omega & \text { circular frequency, } \omega=2 \pi \mathrm{f}\end{array}$

Subscripts:

j quantity evaluated at $j$ th node located at $x=(j-1) \Delta x$

- ambient property

s mean flow property

t throat quantity

Superscripts:

$\mathrm{n}$

quantity evaluated at time $t=n \Delta t$

(1)

predictor quantity

A tilde ( $)$ over a symbol indicates a dimensional variable. A caret (^) over a symbol indicates a small acoustic perturbation.

\section{ANALYSIS}

In the appendix the quasi one-dimensional equations governing acoustic propagation through a variable-area flow duct are developed. The physical system is illustrated in figure 1. An acoustic source located at $x=0(j=1)$ propagates sound upstream (in the positive $x$ direction) against a steady, high subsonic Mach number, isentropic, compressible mean flow. The mean flow, travelling in the negative $x$ direction, accelerates in the throat section as controlled by the area variation $A(x)$.

\section{Acoustics Equations}

The governing propagation equations, derived in the appendix, are as follows:

$$
\frac{\partial \rho}{\partial t}+\frac{\partial}{\partial x}\left(\rho_{s} u+u_{s} \rho+u \rho\right)+\frac{1}{A}\left(\rho_{s} u+u_{s} \rho+u \rho\right) \frac{d A}{d x}=0
$$




$$
\begin{aligned}
& \frac{\partial u}{\partial t}+\frac{\partial}{\partial x}\left\{u s u+\frac{u^{2}}{2}+c_{s}^{2}\left[\frac{\rho}{\rho_{s}}+\frac{\gamma-2}{2}\left(\frac{\rho}{\rho_{s}}\right)^{2}\right]\right\}=0 \\
& p=c_{s}^{2}\left(1+\frac{\gamma-1}{2} \frac{\rho}{\rho_{s}}\right) \rho
\end{aligned}
$$

where subscript $s$ indicates a steady state mean flow quantity. All variables and coefficients in equations (1) to (3) have been nondimensionalized. (See the appendix.) Briefly, density has been nondimensionalized by $\rho_{0}^{\prime}$ velocity by $c_{0}$, pressure by $\rho_{0} c^{2}$, length by multiplying by $k$, and time by multiplying by $\omega$. 'It should also be observed that the equations are nonlinear.

Conservative form.- For the purpose of applying a finite difference scheme, equations (1) and (2) can be expressed in the following conservative form:

$$
\begin{aligned}
& \frac{\partial \rho}{\partial t}+\frac{\partial r}{\partial x}+g=0 \\
& \frac{\partial u}{\partial t}+\frac{\partial s}{\partial x}=0
\end{aligned}
$$

where

$$
\begin{aligned}
& r(x, t)=\rho_{s} u+u_{s} \rho+u \rho \\
& s(x, t)=u_{s} u+\frac{u^{2}}{2}+c_{s}^{2}\left[\frac{\rho}{\rho_{s}}+\frac{\gamma-2}{2}\left(\frac{\rho}{\rho_{s}}\right)^{2}\right] \\
& g(x, t)=\frac{1}{A}\left(\rho_{s} u+u_{s} \rho+u \rho\right) \frac{d A}{d x}
\end{aligned}
$$

Boundary conditions.- A harmonically varying source is assumed at $x=0$

$$
\rho(0, t)=s \sin t \quad(t \geqslant 0)
$$

where the amplitude $S$ is calculated to give a specified source strength as discussed later. A termination condition

$$
p(L, t)-u(L, t)=0
$$


is applied at $\mathrm{x}=\mathrm{L}$ which, for a steady state, harmonically varying solution, reduces to the usual nonreflective impedance condition. Thus, the solutions for $\rho$ and $\mathrm{u}$ are obtained from these equations, and $\rho$ is substituted into equation (3) to get the pressure $p$.

\section{Numerical Method}

A fourth-order MacCormack differencing scheme (ref. 9) is used to generate a numerical solution of equations $(4)$ to $(8)$. The process has two steps applied at $\Delta t / 2$ intervals. The first step involves a backward predictor and a forward corrector (BPFC). The second step uses a forward predictor with a backward corrector $(\mathrm{FPBC})$.

Consider equation (4) for the acoustic density and the discretization model illustrated in figure 1. Let $\rho_{j}^{n}$ be the numerical value of $\rho\left(x_{j}, n \Delta t\right)$, where $j=1,2, \ldots, J$ and $n=0,1,2, \ldots, N$. Assume that the values for $\rho_{j}^{n}$ have been calculated, and it is desired to calculate the corresponding valuss at the next $\Delta t$ time step, that is, $\rho_{j}^{n+1}$.

BPFC phase.- In order to calculate $\rho_{\dot{j}}^{n+1}$, the intermediate values $\rho_{\dot{j}}^{n+(1 / 2)}$ are calculated for $\Delta t / 2$ by using the backward predictor/forward corrector phase of the differencing algorithm. The difference relations are $(j=1,2, \ldots, J)$

$$
\begin{aligned}
& \rho_{j}^{(1)}=\rho_{j}^{n}-\frac{\Delta t / 2}{6 \Delta x}\left(7 r_{j}^{n}-8 r_{j-1}^{n}+r_{j-2}^{n}\right)-\frac{\Delta t}{2} g_{j}^{n} \\
& \rho_{j}^{n+(1 / 2)}=\frac{1}{2}\left[\rho_{j}^{n}+\rho_{j}^{(1)}+\frac{\Delta t / 2}{6 \Delta x}\left(7 r_{j}^{(1)}-8 r_{j+1}^{(1)}+r_{j+2}^{(1)}\right)-\frac{\Delta t}{2} g_{j}^{(1)}\right]
\end{aligned}
$$

The predictor quantities are denoted by a superscript (1), for example, $\rho_{j}^{(1)}$. Flux and force values based on predictor quantities are denoted in a similar manner, for example, $r_{j}^{(1)}$ and $g_{j}^{(1)}$. (See eqs. (6) and (8).)

The predictor values $\rho_{j}^{(1)}$ at the next $\Delta t / 2$ are computed from equation (11). Since $j=1,2, \ldots, J$, this requires flux values $r_{0}$ and $r_{-1}$ at the two extra grid points ahead of the source plane $(j=1)$. These are computed using third-order backward extrapolation as follows:

$$
\begin{gathered}
r_{0}^{n}=4 r_{1}^{n}-6 r_{2}^{n}+4 r_{3}^{n}-r_{4}^{n} \\
r_{-1}^{n}=4 r_{0}^{n}-6 r_{1}^{n}+4 r_{2}^{n}-r_{3}^{n}
\end{gathered}
$$


The predictor values $\rho_{j}^{(1)}$ are used in equation (12) to calculate the corresponding corrector values $\rho_{j}^{n+(1 / 2)}$ at the $n \Delta t+(\Delta t / 2)$ time step.

FPBC phase.- The next step is to use the corrector values $\rho_{j}^{n+(1 / 2)}$ at $n \Delta t \overline{+(\Delta t / 2)}$ in the forward predictor/backward corrector phase of the scheme to calculate $\rho_{j}^{n}$ at $n \Delta t+\Delta t$. The required relations are $(j=1,2, \ldots, J)$

$$
\begin{aligned}
& \rho_{j}^{(1)}=\rho_{j}^{n+(1 / 2)}+\frac{\Delta t / 2}{6 \Delta x}\left(7 r_{j}^{n+(1 / 2)}-8 r_{j+1}^{n+(1 / 2)}+r_{j+2}^{n+(1 / 2)}\right)-\frac{\Delta t}{2} g_{j}^{n+(1 / 2)} \\
& \rho_{j}^{n+1}=\frac{1}{2}\left[\rho_{j}^{n+(1 / 2)}+\rho_{j}^{(1)}-\frac{\Delta t / 2}{6 \Delta x}\left(7 r_{j}^{(1)}-8 r_{j-1}^{(1)}+r_{j-2}^{(1)}\right)-\frac{\Delta t}{2} g_{j}^{(1)}\right]
\end{aligned}
$$

The predictor values $\rho_{j}^{(1)}$ in this case require flux values $r_{J+1}$ and $r_{J+2}$ at the two extra grid points beyond the termination plane $(j=J)$. These are calculated using third-order forward extrapolation of the variables $\rho_{j}^{n+(1 / 2)}$ from the BPFC
step. These relations are as follows:

$$
\begin{aligned}
& r_{J+1}^{(1)}=4 r_{J}^{(1)}-6 r_{J-1}^{(1)}+4 r_{J-2}^{(1)}-r_{J-3}^{(1)} \\
& r_{J+2}^{(1)}=4 r_{J+1}^{(1)}-6 r_{J}^{(1)}+4 r_{J-1}^{(1)}-r_{J-2}^{(1)}
\end{aligned}
$$

The same type of differencing relations (eqs. (11) to (18)) is applied to equations (5) and (7) in order to compute the acoustic particle velocity $\mathrm{u}_{j}^{\mathrm{n}}$. Since it is a straightforward matter to replace $\rho_{j}^{n}$ by $u_{j}^{n}$, these equations are not given herein. Once the solutions for $\rho_{j}^{n}$ and $u_{j}^{n}$ have been obtained, $p_{j}^{n}$ is calculated
from equation (3).

Numerical boundary conditions.- In order to satisfy the source (eq. (9)) and termination (eq. (10)) boundary conditions, the characteristic variables $\rho / \rho_{s}+u / c_{s}$ (for right-moving waves) and $\rho / \rho_{s}-u / c_{s}$ (for left-moving waves) are used. For example, at any time $t$ the instantaneous'density fluctuation at the source $(x=0)$ is given by equation (9). Thus, for the finite discretization, the source boundary condition is

$$
\rho_{1}=s \sin t
$$


The amplitude $S$ is determined so that the pressure level at the source peaks at the desired decibel level (reference $2.0 \times 10^{-5} \mathrm{~N} / \mathrm{m}^{2}$ ). In the FPBC and BPFC sections of the difference algorithm, the quantity

$$
T_{1}=\frac{\rho_{1}}{\rho_{s 1}}-\frac{u_{1}}{c_{s 1}}
$$

is calculated at a previous time step where $\rho_{1}=\rho(0, t), u_{1}=u(0, t), \rho_{s 1}=\rho_{s}(0)$ and $c_{s 1}=c_{s}(0)$. Therefore, at any time $t$ the source density is given by equation (9), and the corresponding acoustic particle velocity is obtained from equation (19); that is,

$$
u_{1}=c_{s 1}\left(\frac{\rho_{1}}{\rho_{s 1}}-T_{1}\right)
$$

At the termination section $\mathrm{x}=\mathrm{L}$, a nonreflective boundary condition is imposed. For a harmonic steady state this requires that the impedance of an outgoing wave be unity or, in other words, that $p=u$ (eq. (10)). The required boundary conditions are obtained by calculating

$$
T_{2}=\frac{\rho_{\mathrm{J}}}{\rho_{\mathrm{sJ}}}+\frac{\mathrm{u}_{\mathrm{J}}}{\mathrm{c}_{\mathrm{sJ}}}
$$

at a previous time step. This value for $T_{2}$ together with equation (10) and the linearized form of equation (3) yields the following boundary relations for $\rho_{J}$ and $\mathrm{u}_{\mathrm{J}}$ :

$$
\begin{aligned}
& \rho_{J}=\frac{T_{2}}{c_{s J}+\left(1 / \rho_{s J}\right)} \\
& u_{j}=\frac{c_{s J}^{2} T_{s}}{c_{s J}+\left(1 / \rho_{s J}\right)}
\end{aligned}
$$

where $\rho_{J}=\rho(L, t), u_{J}=u(L, t), \quad \rho_{S J}=\rho_{S}(L)$, and $c_{S J}=c_{S}(L)$. Note that the values of $T_{1}$ and $T_{2}$ are computed at time $[n-(1 / 2)] \Delta t .{ }^{S}$ The physical boundary conditions, however, are imposed at time $n \Delta t$. 
The finite difference analysis discussed previously is applied in this section to a variable area duct containing high-speed subsonic flow and a high-intensity propagating acoustic wave.

\section{Duct Geometry}

The duct configuration is sketched in figure 1 and has an overall length of about $1.518 \mathrm{~m}$. The two uniform sections on each end of the duct have a square cross section $0.0508 \mathrm{~m}$ on each side. The width of the duct is maintained constant $(0.0508 \mathrm{~m})$ as the height is gradually reduced to give a $2 / 1$ area reduction at the throat $(x / L=0.50)$. Thus, the throat is rectangular $(0.0508 \times 0.0254 \mathrm{~m})$.

The duct contour was designed so that a linear Mach number variation is achieved when the inlet $(x / L=0.25)$ and throat $(x / L=0.50)$ Mach numbers are 0.3 and 1.0 , respectively. With this flow distribution, the one-dimensional gas dynamics equations (eqs. (A1) to (A3)) can be solved to yield a closed form expression for the corresponding area variation $A(x)$. For a uniform section

$$
A(x)=2.035
$$

$$
\left(\left|\frac{2 x}{L}-1\right|>\frac{1}{2}\right)
$$

and for the variable area section

$$
A(x)=\frac{1}{G(x)}\left[\frac{\gamma+1}{2}-\frac{\gamma-1}{2} G^{2}(x)\right]^{\frac{1}{\gamma-1}} \quad\left(\left|\frac{2 x}{L}-1\right| \leqslant \frac{1}{2}\right)
$$

where $G(x)$ is given by

$$
G(x)=1-1.34857\left|\frac{2 x}{L}-1\right|
$$

\section{Sample Calculation}

In order to illustrate the finite difference algorithm, a calculation for the mean flow distribution shown in figure 2 is presented. Here the mean throat Mach number was arbitrarily set at 0.85 . The distribution was then calculated by solving the one-dimensional gas dynamics equations using the area variation $A(x)$ given by equations (24) to (26). The Mach number increases from about 0.28 at $\mathrm{x} / \mathrm{L}=0.25$ to 0.85 at the throat and then drops to about 0.28 at $x / L=0.75$. Hence, the calculated mean flow is symmetric about the throat $(x / L=0.50)$. For the acoustic data which follow, a negative throat Mach number is indicated $\left(M_{t}=-0.85\right)$ to emphasize 
that the flow is in the negative $x$ direction, opposite to the propagation of acoustic waves from the source. This corresponds to the aeroengine inlet situation.

Figure 3 shows the acoustic pressure distribution calculated by the nonlinear finite difference algorithm (eqs. (11) to (23)) for a 135-dB acoustic source oscillating at $500 \mathrm{~Hz}$. For comparison, the results of a linear finite element calculation are also shown. The finite element algorithm is based on the Galerkin/weightedresidual method using one-dimensional, isoparametric, Hermite elements of third order (see ref. 11, for example).

Owing to the nonlinear character of the finite difference model, the solid curve in figure 3 is actually the fundamental $(500 \mathrm{~Hz}$ ) component ( $\sin t)$ of the acoustic pressure. The two calculations, nonlinear finite difference and linear finite element, are in close agreement. This is unexpectedly true in the high-intensity throat region. Spectral data are also presented in table I for the fundamental and first three harmonics of the nonlinear finite difference pressure calculation. Comparison of these data indicates that a considerable amount of energy is shifted into the higher harmonics. For example, at $\mathrm{x} / \mathrm{L}=1.00$, the amount of energy contained by the first harmonic ( $\sin 2 t$ ) is about 16.5 percent of that contained in the fundamental.

A plot of the acoustic particle velocity is shown in figure 4, and the acoustic streaming velocity component is presented in figure 5. With a linear acoustics model driven sinusoidally, the acoustic velocity would have zero mean. With nonlinearities present, however, the acoustic velocity exhibits, in addition to higher harmonics, a nonzero mean component termed the streaming velocity. A nonzero streaming velocity indicates a transfer of acoustical energy to the mean flow. As shown in figure 5, the acoustic streaming velocity for the present case peaks in the throat region, but compared with the mean flow (fig. 2), is quite small.

Figure 6 shows time histories of the acoustic pressure at five axial locations after steady state was achieved by the finite difference algorithm. Figure 6(a), shown for reference, is the source pressure time history, which varies sinusoidally at $500 \mathrm{~Hz}$ (sin $t$ ). The peak amplitude corresponds to a level of $135 \mathrm{~dB}$. At station $\mathrm{x} / \mathrm{L}=0.25$ (fig. 6(b)), which is located at the beginning of the area variation, the pressure response is still very close to a pure sine wave. For example, table I shows a first harmonic level of $107.1 \mathrm{~dB}$ or about $27.7 \mathrm{~dB}$ lower than the fundamental $(134.8 \mathrm{~dB})$.

At the throat (fig. $6(c), x / L=0.50$ ), the pressure shows appreciable distortion from a pure sine wave. Here the pressure peaks at about $150.1 \mathrm{~dB}$ (table $\mathrm{I}$ and fig. 3). The results of the linear finite element indicate a peak of approximately $150.0 \mathrm{~dB}$. The first harmonic $(137.4 \mathrm{~dB})$ in this instance is only about $12.7 \mathrm{~dB}$ lower than the fundamental $(150.1 \mathrm{~dB})$. It thus appears, by virtue of the increased harmonic content, that the nonlinearities are becoming more important, although the linear solution still adequately predicts the peak level in the throat region.

Further upstream at the end of the area variation $(x / L=0.75)$, as shown in figure 6(d), considerable harmonic distortion remains although the peak level has dropped rapidly to about $134.6 \mathrm{~dB}$. Here the first harmonic (126.5 dB) is only about 8.1 dB lower than the fundamental $(134.6 \mathrm{~dB})$. Finally, at the termination (fig. $6(e), x / L=1.00$ ), about the same picture prevails, although the beginning of a steepening in the wave is noticeable. 
This wave steepening behavior is examined further in figure 7, where the source strength is varied from 134 to $137 \mathrm{~dB}$. Here a very definite nonlinear steepening appears in the pressure time history at the termination. This indicates that by increasing the source strength, the acoustic response approaches a shock condition.

\section{Comparisons with Asymptotic Theory}

As discussed previously, the finite difference algorithm was compared with a linear finite element solution for the classical small amplitude linear acoustics case. Here a check is made for a typical nonlinear situation by comparing the finite difference solution with the asymptotic theory of Callegari and Myers (ref. 10). The typical nonlinear situation arises at higher sound pressure levels for throat Mach numbers approaching unity. In this theory $\left(1-\left|M_{t}\right|\right)$ is taken as the (small) perturbation parameter.

A slightly different geometry was used for these calculations. As shown in figure 8 , the constant area entrance and termination sections were eliminated, and the area variation is given by equation (25) where

$$
G(x)=1-0.91287\left|\frac{x}{L}-\frac{1}{2}\right| \quad\left(0 \leqslant \frac{x}{L} \leqslant 1\right)
$$

For this geometry, the area reduction ratio is about $1.32 / 1$, so that for an entrance Mach number of about 0.51 , the flow accelerates linearly to a sonic velocity at the throat. Hence, this area variation provides a much more gradual choking of the flow than does the configuration (eqs. (25) and (26)) used in the earlier calculations.

Results are presented in figures 9 to 11 for three combinations of source strength and throat Mach number. In each case, the acoustic velocity divided by $\left(1-\left|M_{t}\right|\right)^{2}$ at $x=0.75 L$ and $x=L$ is shown after steady state had been achieved by the differencing algorithm. For the cases presented, the quantity $\left(1-\left|M_{t}\right|\right)^{2}$ is of the order of $10^{-2}$ or less. Hence, the calculations are accurate to at least three decimal places. The solid lines in figures 9 to 11 indicate the finite difference solution. The circular and square symbols indicate the calculated values based on the asymptotic theory of Callegari and Myers (ref. 10).

The comparisons for a throat Mach number of $M_{t}=-0.90$ are graphed in figures 9 and 10. The strengths, for an equivalent pressure source located at $\mathrm{x}=0$ (fig. 8), are 130 and $140 \mathrm{~dB}$, respectively. In both cases, no shocks were predicted or, for that matter, were expected, and the finite difference calculation agrees very well with the asymptotic theory. At $140 \mathrm{~dB}$, as shown in figure 10 , a severely distorted time history occurs at $\mathrm{x} / \mathrm{L}=0.75$, and the agreement is excellent.

Figure 11 illustrates a similar comparison for a 130-dB source when the throat Mach number is increased to $\mathrm{M}_{t}=-0.95$. This situation is very close to shock formation (ref. 10). Here the comparison is still very good. However, there are some minor differences between the asymptotic theory and the finite difference algorithm near $t \approx \pi / 2$ for $x / L=0.75$. 
For this latter case (fig. 11), a further increase in the throat Mach number (or an increase in the source strength) will precipitate a shock and a resulting loss of acoustical energy. For weak shocks, the energy relation given by equation (3) might suffice. Stronger shocks, manifested by appreciable loss in acoustical energy and increase in entropy, will require that the general energy equation (ref. 12) be substituted in lieu of equation (3). In both instances a "shock" jump condition must be added to the finite difference code.

Finally, it should be noted that the asymptotic theory of reference 10 is extremely accurate when $\left(1-\left|M_{t}\right|\right)$ approaches zero; that is, when the throat Mach number approaches unity. It is precisely these situations which present the most computational difficulty for the finite difference algorithm, since axial wavelengths become increasingly small, which necessitates extremely fine time/space meshes. Therefore, the comparisons presented in figures 9 and 10 are thought to represent a stringent test of the differencing procedure to simulate the nonlinear acoustics case. The finite difference algorithm is more effective in handling the calculations for larger values of $\left(1-\left|M_{t}\right|\right)$. With these situations the asymptotic theory is less accurate. Hence, these two independent methods nicely complement one another.

\section{CONCLUDING REMARKS}

An explicit finite difference algorithm based on a MacCormack scheme was used to solve numerically the one-dimensional nonlinear acoustic equations for a variable area duct. The procedure iterated over the time/space discretization grid until a steady state solution was achieved. Sample calculations were presented for several combinations of area reduction ratio, throat Mach number, and source sound pressure level.

The principal effect of the nonlinearities was to shift acoustical energy to higher harmonics. With increased source strengths, wave steepening was observed. This phenomenon suggests that the acoustical response may approach a shock behavior at a higher sound pressure level as the throat Mach number approaches sonic value. Where applicable, comparisons were made with the calculations from a linear finite element algorithm. On a peak level basis, good agreement between the nonlinear finite difference and linear finite element solutions was observed, even though a peak sound pressure level of about $150 \mathrm{~dB}$ occurred in the throat region. Nonlinear, steady state waveform solutions were shown to be in excellent agreement with a nonlinear asymptotic theory.

Langley Research Center National Aeronautics and Space Administration Hampton, VA 23665

June 9, 1983 


\section{APPENDIX}

DERIVATION OF NONLINEAR ACOUSTIC EQUATIONS

In the following, the one-dimensional compressible fluid continuum equations are used to derive the governing acoustic propagation relations.

\section{Fluid Continuum Equations}

The one-dimensional compressible flow equations for an isentropic fluid may be expressed in terms of the density $\rho$ and velocity $u$ (ref. 12). The equation of continuity is

$$
\frac{\partial \tilde{\rho}}{\partial \tilde{t}}+\frac{\partial}{\partial \tilde{x}}(\tilde{\rho} \tilde{u})+\frac{\tilde{\rho} \tilde{u}}{\tilde{A}} \frac{d \tilde{A}}{d \tilde{x}}=0
$$

and the balance of momentum is given by

$$
\frac{\partial \tilde{u}}{\partial \tilde{t}}+\frac{\partial}{\partial \tilde{x}}\left(\frac{\tilde{u}^{2}}{2}+\frac{\gamma}{\gamma-1} \frac{p_{o}}{\rho_{o}} \tilde{\rho} \gamma-1\right)=0
$$

The pressure is determined from

$$
\tilde{\mathrm{p}}=\frac{\mathrm{p}_{0}}{\rho_{0}^{\gamma}} \tilde{\rho}^{\gamma}
$$

In the above equations $p_{0}$ and $\rho_{0}$ are the ambient pressure and density, respectively.

\section{Acoustic Perturbations}

$$
\begin{aligned}
& \text { In equations }(A 1) \text { to (A3) let } \\
& \left.\begin{array}{r}
\tilde{\rho}(\tilde{x}, \tilde{t})=\tilde{\rho}_{S}(\tilde{x})+\varepsilon \hat{\rho}(\tilde{x}, \tilde{t}) \\
\tilde{u}(\tilde{x}, \tilde{t})=\tilde{u}_{s}(\tilde{x})+\varepsilon \hat{u}(\tilde{x}, \tilde{t}) \\
\tilde{p}(\tilde{x}, \tilde{t})=\tilde{p}_{S}(\tilde{x})+\varepsilon \hat{p}(\tilde{x}, \tilde{t})
\end{array}\right)
\end{aligned}
$$


where a caret $\left({ }^{\wedge}\right)$ over a symbol indicates a small acoustic perturbation about the basic steady flow state, denoted by a subscript $s$. The steady state flow equations are

$$
\begin{aligned}
& \frac{\partial}{\partial \tilde{x}}\left(\tilde{u}_{s} \tilde{\rho}_{S}\right)+\frac{\tilde{\rho}_{S} \tilde{u}_{S}}{\tilde{A}} \frac{d \tilde{A}}{d \tilde{x}}=0 \\
& \frac{\partial}{\partial \tilde{x}}\left(\frac{\tilde{u}_{S}^{2}}{2}+\frac{\gamma}{\gamma-1} \frac{p_{O}}{\rho_{0}^{\gamma}} \tilde{\rho}_{S}^{\gamma-1}\right)=0 \\
& \tilde{p}_{S}=\frac{p_{0}}{\rho_{0}^{\gamma}} \tilde{\rho}_{S}^{\gamma}
\end{aligned}
$$

Substituting equations ( $A 4$ ) into equations ( $A 1$ ) to ( $A 3$ ) and expanding and then subtracting steady state terms (eqs. (A5) to (A7)) gives the final nonlinear acoustic equations

$$
\begin{aligned}
& \frac{\partial \hat{\rho}}{\partial \tilde{t}}+\frac{\partial}{\partial \tilde{x}}\left(\tilde{\rho}_{S} \hat{u}+\tilde{u}_{s} \hat{\rho}+\varepsilon \hat{u} \hat{\rho}\right)+\frac{1}{\tilde{A}}\left(\tilde{\rho}_{S} u+\tilde{u}_{s} \hat{\rho}+\varepsilon \hat{u} \hat{\rho}\right) \frac{d \tilde{A}}{d \tilde{x}}=0 \\
& \frac{\partial \tilde{u}}{\partial \tilde{t}}+\frac{\partial}{\partial \tilde{x}}\left\langle\tilde{u}_{s} \hat{u}+\frac{\varepsilon u^{2}}{2}+\tilde{c}_{s}^{2}\left[\frac{\hat{\rho}}{\tilde{\rho}_{S}}+\varepsilon \frac{\gamma-2}{2}\left(\frac{\hat{\rho}}{\tilde{\rho}_{S}}\right)^{2}\right]\right\}=0
\end{aligned}
$$

The acoustic pressure is given by the relation

$$
\hat{p}=\tilde{c}_{S}^{2}\left(1+\varepsilon \frac{\gamma-1}{2} \frac{\hat{\rho}}{\tilde{\rho}_{S}}\right) \rho
$$

where the local speed of sound is given by

$$
\tilde{c}_{S}^{2}=\frac{\gamma \tilde{p}_{S}}{\tilde{\rho}_{S}}=\frac{\gamma p_{0}}{\rho_{0}^{\gamma}} \tilde{\rho}_{S}^{\gamma-1}
$$




\section{APPENDIX}

The parameter $\varepsilon$ denotes a nonlinear acoustic term. With $\varepsilon=0$, equations (A8) to (A10) give the usual one-dimensional linear acoustic solution. Whereas with $\varepsilon=1$, a nonlinear solution is obtained.

\section{Nondimensional Acoustic Equations}

Equations ( $A 8)$ to $(A 10)$ are nondimensionalized by using the following transformations:

$$
\begin{aligned}
& \rho(x, t)=\frac{\hat{\rho}(\tilde{x}, \tilde{t})}{\rho_{O}} \\
& u(x, t)=\frac{\hat{u}(\tilde{x}, \tilde{t})}{c_{O}} \\
& p(x, t)=\frac{\hat{p}(\tilde{x}, \tilde{t})}{\rho_{O} c_{0}} \\
& x=k \tilde{x} \\
& k=\frac{\omega}{c_{O}} \\
& c_{S}(x)=\frac{\tilde{u_{S}}(\tilde{x})}{c_{O}} \\
& \rho_{S}(x)=\frac{\tilde{\rho_{S}}(\tilde{x})}{\rho_{O}} \\
& c_{O} \\
& =\frac{\tilde{A}(\tilde{x})}{A_{t}}
\end{aligned}
$$


Substituting equations (A12) into equations (A8) to (A10) yields the nonlinear acoustic equations in a final nondimensional form

$$
\begin{aligned}
& \frac{\partial \rho}{\partial t}+\frac{\partial}{\partial x}\left(\rho_{s} u+u_{s} \rho+\varepsilon u \rho\right)+\frac{1}{A}\left(\rho_{s} u+u_{s} \rho+\varepsilon u \rho\right) \frac{d A}{d x}=0 \\
& \frac{\partial u}{\partial t}+\frac{\partial}{\partial x}\left\langle u_{s} u+\varepsilon \frac{u^{2}}{2}+c_{s}^{2}\left[\frac{\rho}{\rho_{s}}+\varepsilon \frac{\gamma-2}{2}\left(\frac{\rho}{\rho_{s}}\right)^{2}\right]\right)=0 \\
& p=c_{s}^{2}\left(1+\varepsilon \frac{\gamma-1}{2} \frac{\rho}{\rho_{s}}\right) \rho \\
& c_{s}^{2}=\frac{r p_{s}}{\rho_{s}}
\end{aligned}
$$

In terms of these nondimensional variables, the instantaneous local Mach number M is

$$
M=\frac{u_{s}+\varepsilon u}{\left(\rho_{s}+\varepsilon \rho\right)^{\frac{\gamma-1}{2}}}
$$




\section{REFERENCES}

1. Abrahamson, A. Louis: ADAM - An Axisymmetric Duct Aeroacoustic Modeling System. NASA CR-3668, 1983.

2. Baumeister, Kenneth J.: Numerical Techniques in Linear Duct Acoustics - 1980-81 Update. NASA TM-82730, 1981.

3. Baumeister, Kenneth J.: Time-Dependent Difference Theory for Noise Propagation in a Two-Dimensional Duct. AIAA J., vol. 18, no. 12, Dec. 1980, pp. $1470-1476$.

4. Baumeister, K. J.: A Time Dependent Difference Theory for Sound Propagation in Ducts with Flow. Paper presented at 98th Acoustical Society of America Meeting (Salt Lake City, Utah), Nov. 26-30, 1979.

5. Baumeister, K. J.: Time Dependent Difference Theory for Sound Propagation in Axisymmetric Ducts with Plug Flow. AIAA-80-1017, June 1980.

6. Myers, M. K.; and Callegari, A. J.: Nonlinear Theory of Shocked Sound Propagation in a Nearly Choked Duct Flow. NASA CR-3549, 1982.

7. Nayfeh, A. H.; Kelly, J. J.; and watson, L. T.: Non-Linear Propagation in Near Sonic Flows. J. Sound \& Vib., vol. 75, no. 3, Apr. 8, 1981, pp. 359-370.

8. Jones, Michael Glen: An Experimental Investigation of Sound Attenuation in a High-Subsonic Mach Number Inlet. M.S. Thesis, The George Washington Univ., Feb. 22, 1982 .

9. Turkel, Eli: On the Practical Use of High order Methods for Hyperbolic Systems. Rep. No. 78-19 (Contracts NAS1-14101 and EY-76-C-02-3077), Inst. for Computer Appl. in Sci. and Eng., Nov. 16, 1578.

10. Callegari, A. J.; and Myers, M. K.: Sound Transmission in Ducts Containing Nearly Choked Flows. AIAA Paper 79-0623, Mar. 1979.

11. Lester, Harold C.; and Parrott, Tony L.: Comparison of Measured and Predicted Impedance at Grazing Incidence. AIAA J., vol. 18, no. 5, May 1980, pp. 504-508.

12. Shapiro, Ascher H.: The Dynamics and Thermodynamics of Compressible Fluid Flow. Vol. II. The Ronald Press Co., c.1954. 
TABLE I.- ACOUSTIC PRESSURE FOR 135-dB SOURCE

$$
\left[M_{t}=0.85 ; f=500 \mathrm{~Hz}\right]
$$

\begin{tabular}{|c|c|c|c|c|c|}
\hline \multirow{2}{*}{$\frac{x}{L}$} & \multicolumn{4}{|c|}{ Nonlinear finite difference, $\mathrm{dB}$} & \multirow{2}{*}{$\begin{array}{l}\text { Linear } \\
\text { finite } \\
\text { element } \\
\mathrm{dB}\end{array}$} \\
\hline & Fundamental & 1st harmonic & 2d harmonic & 3d harmonic & \\
\hline 0.00 & 135.0 & & & & 135.0 \\
\hline .25 & 134.8 & 107.1 & 84.7 & 90.3 & 134.7 \\
\hline .50 & 150.1 & 137.4 & 127.9 & 102.2 & 150.0 \\
\hline .75 & 134.6 & 126.5 & 116.9 & 105.6 & 135.2 \\
\hline 1.00 & 134.7 & 126.9 & 117.1 & 105.4 & 135.3 \\
\hline
\end{tabular}




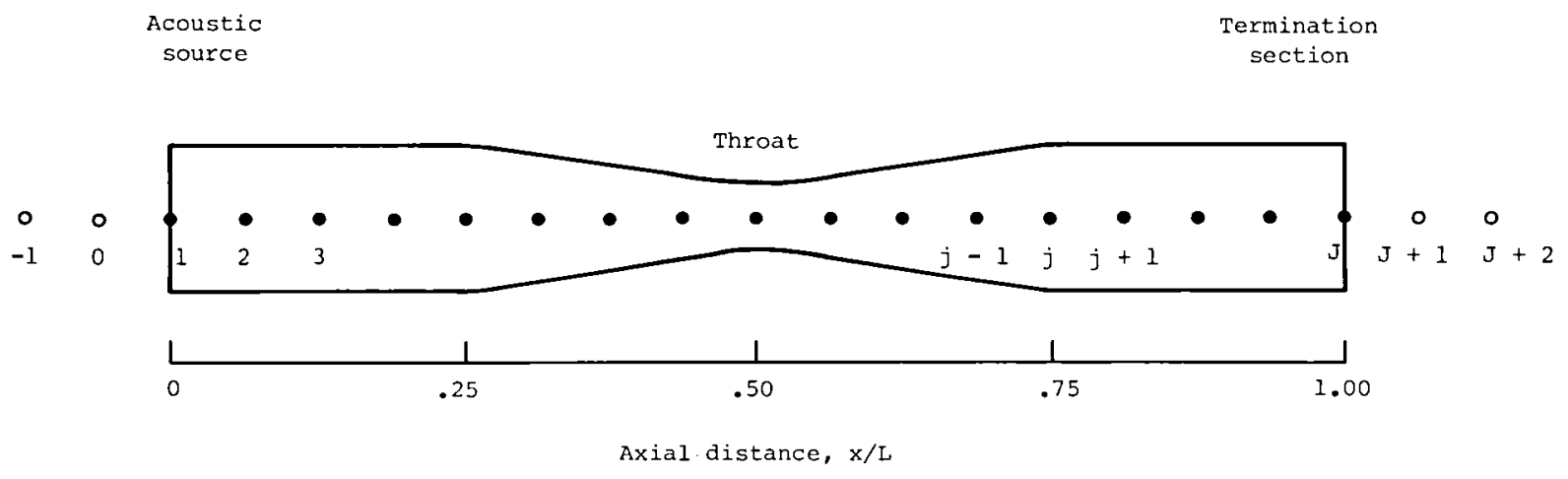

Figure 1.- Variable area flow duct.

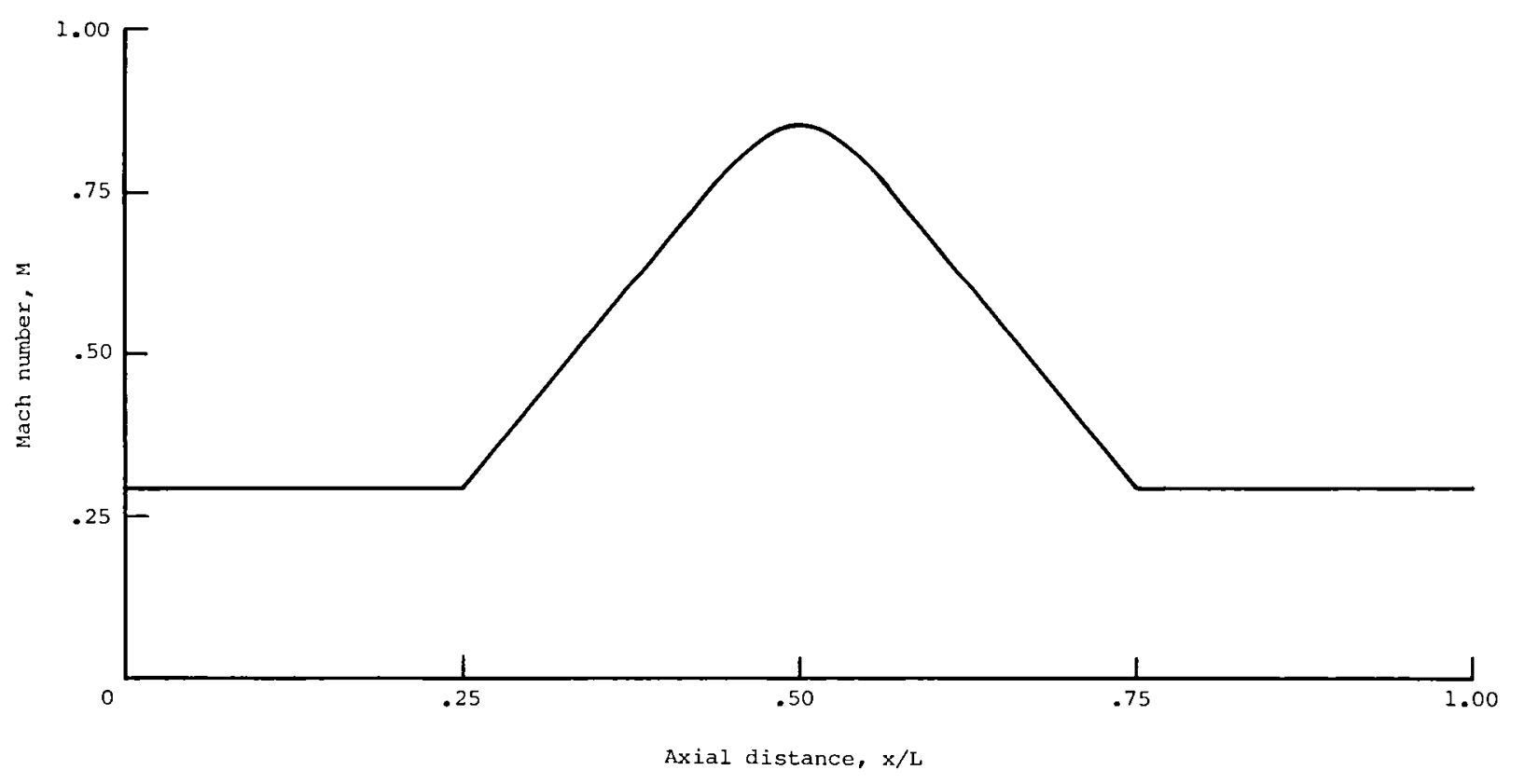

Figure 2.- Mean flow Mach number. $M_{t}=0.85$. 


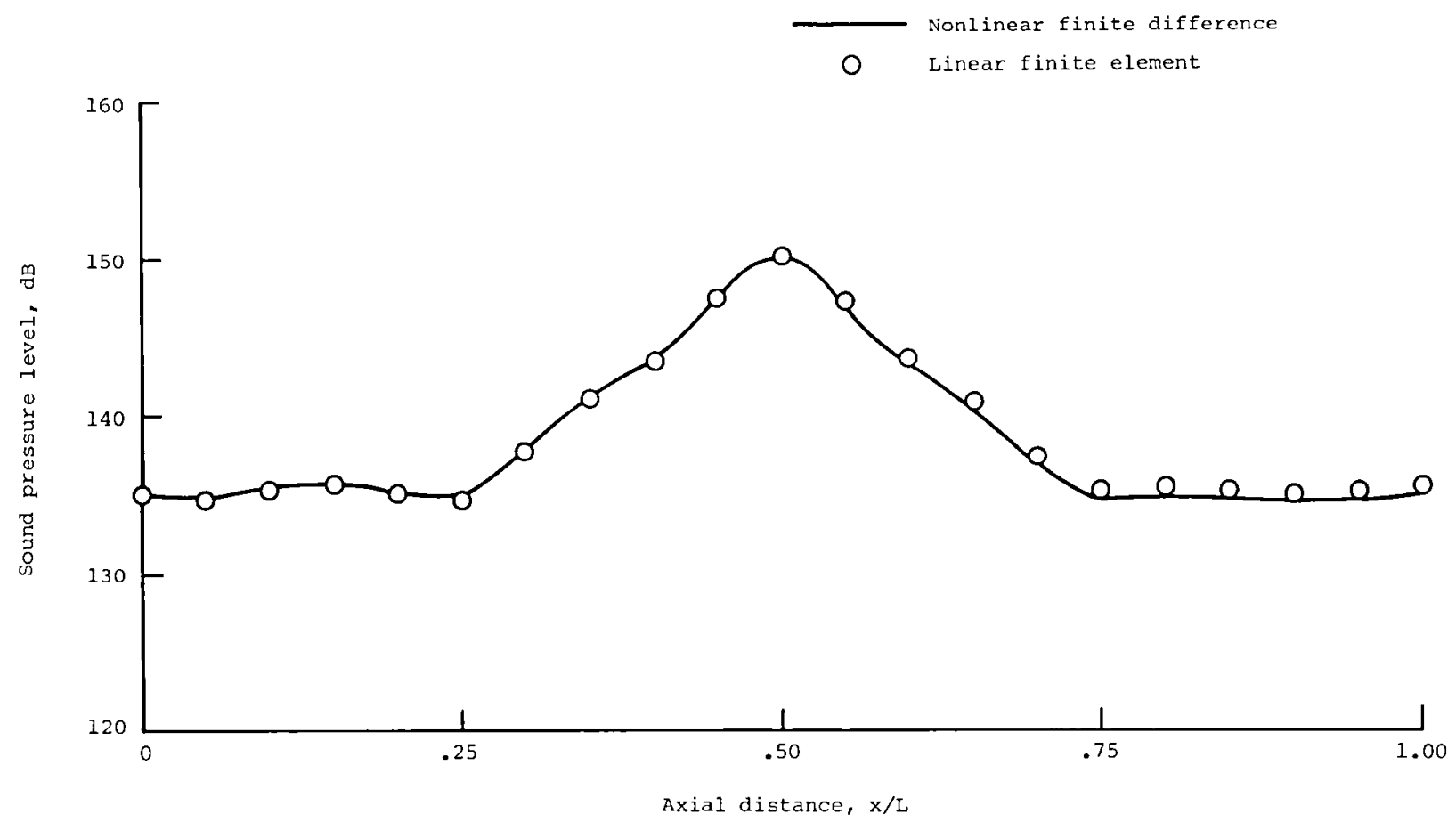

Figure 3.- Acoustic pressure. 135-dB source; $M_{t}=-0.85 ; f=500 \mathrm{~Hz}$.

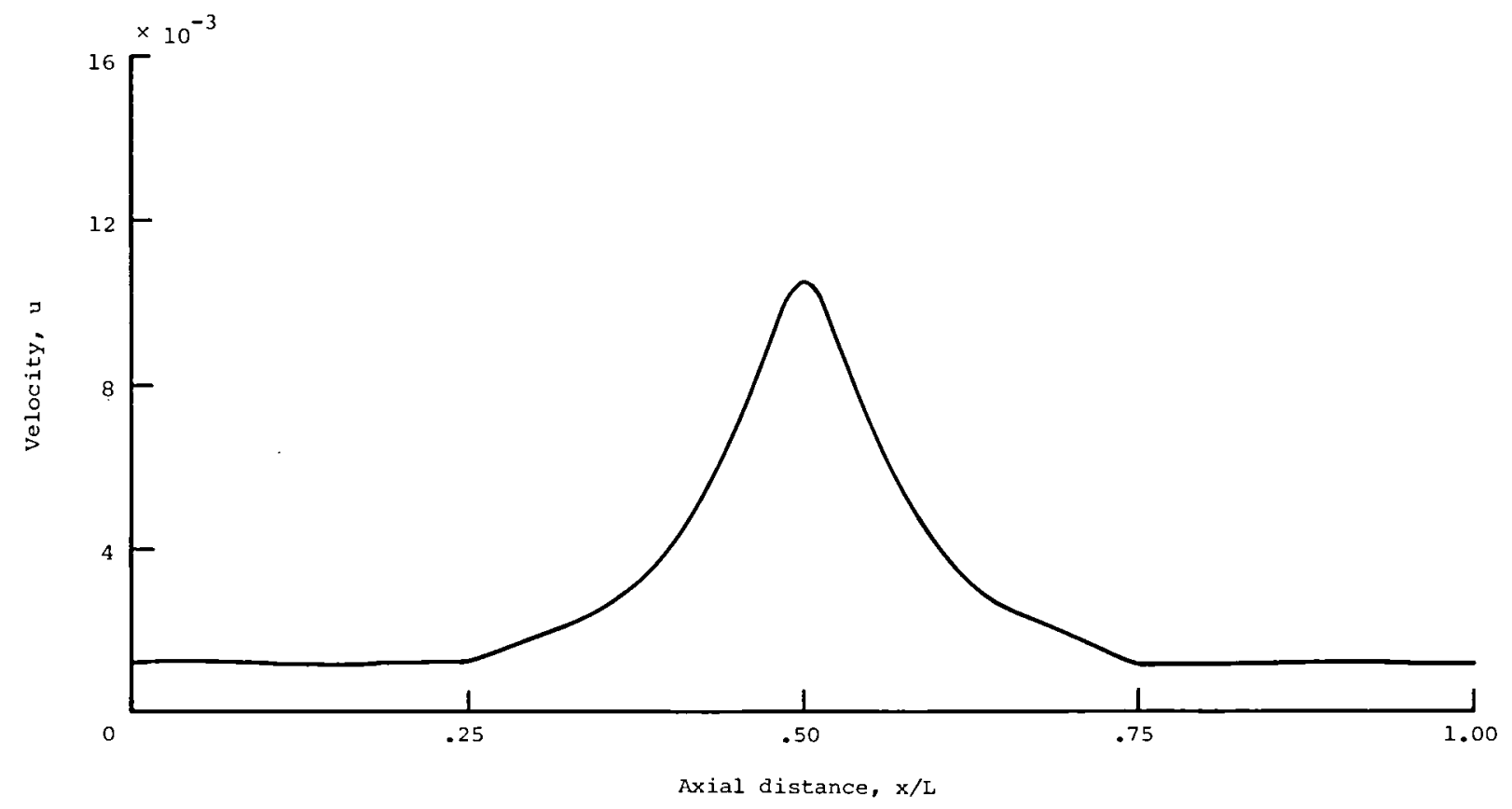

Figure 4.- Acoustic particle velocity. $135-\mathrm{dB}$ source; $\mathrm{M}_{t}=-0.85 ; \mathrm{f}=500 \mathrm{~Hz}$. 


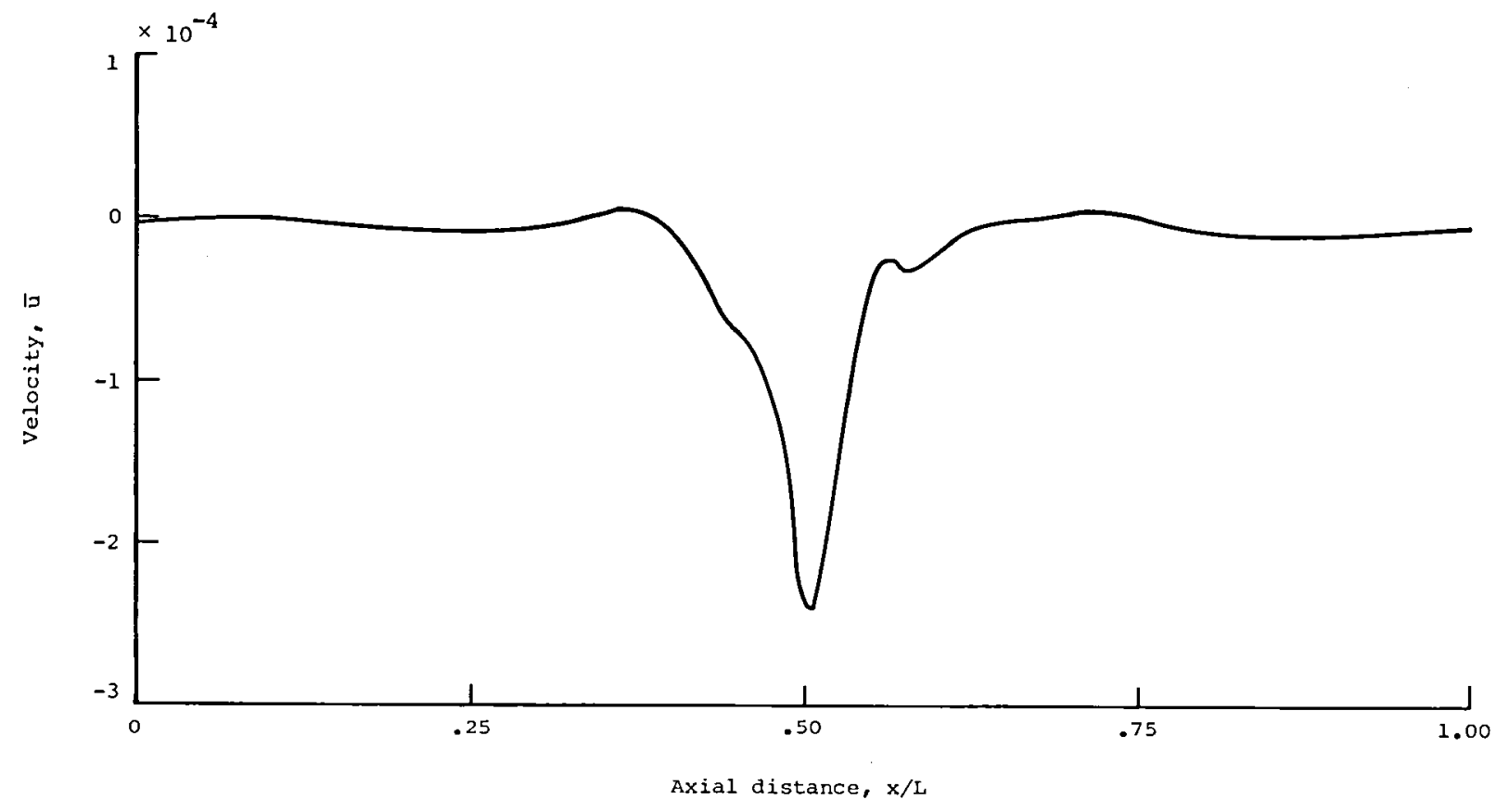

Figure 5.- Acoustic streaming velocity. $135-\mathrm{dB}$ source; $M_{t}=-0.85 ; \quad f=500 \mathrm{~Hz}$. 


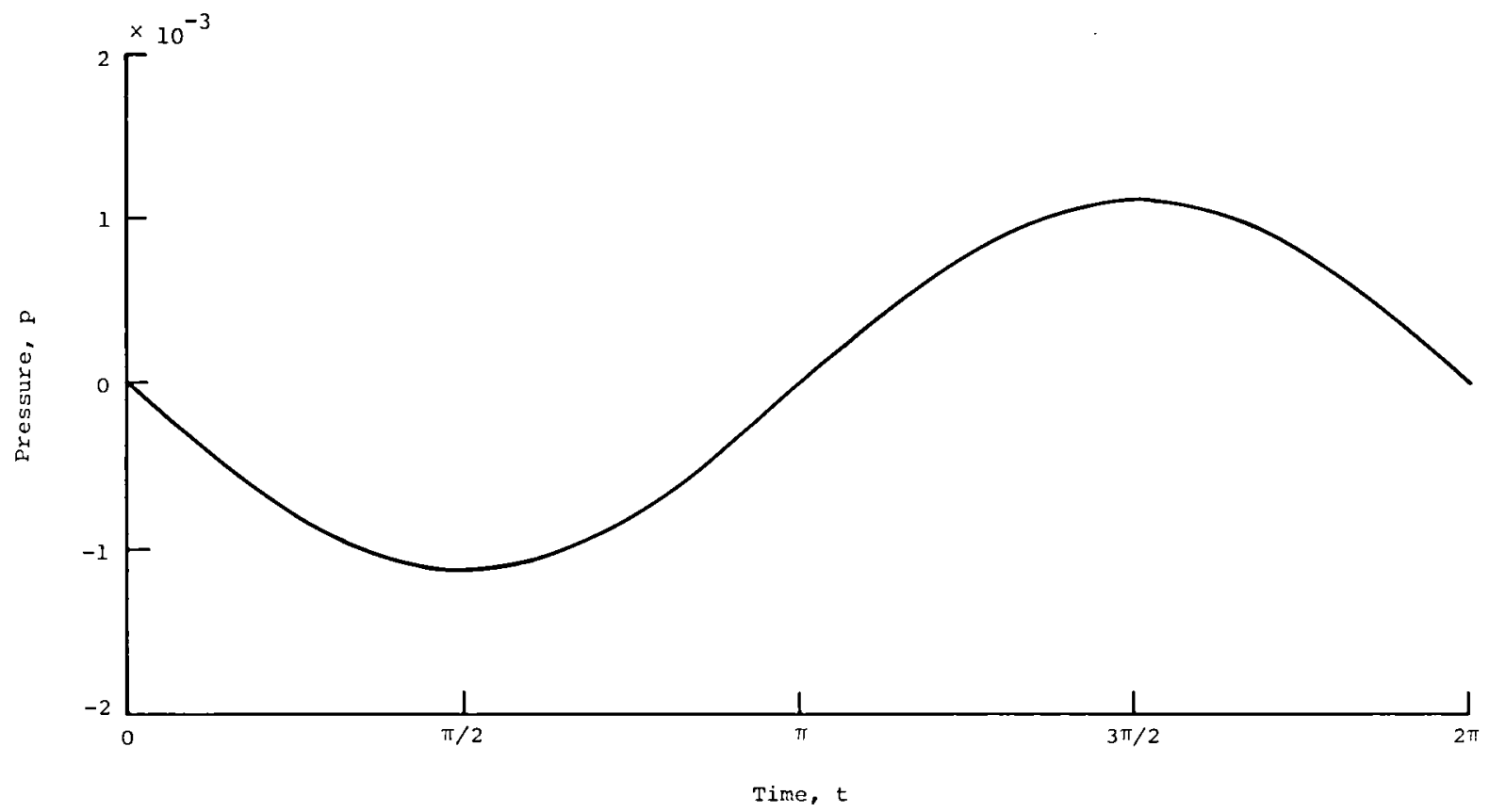

(a) Source; $x / L=0$.

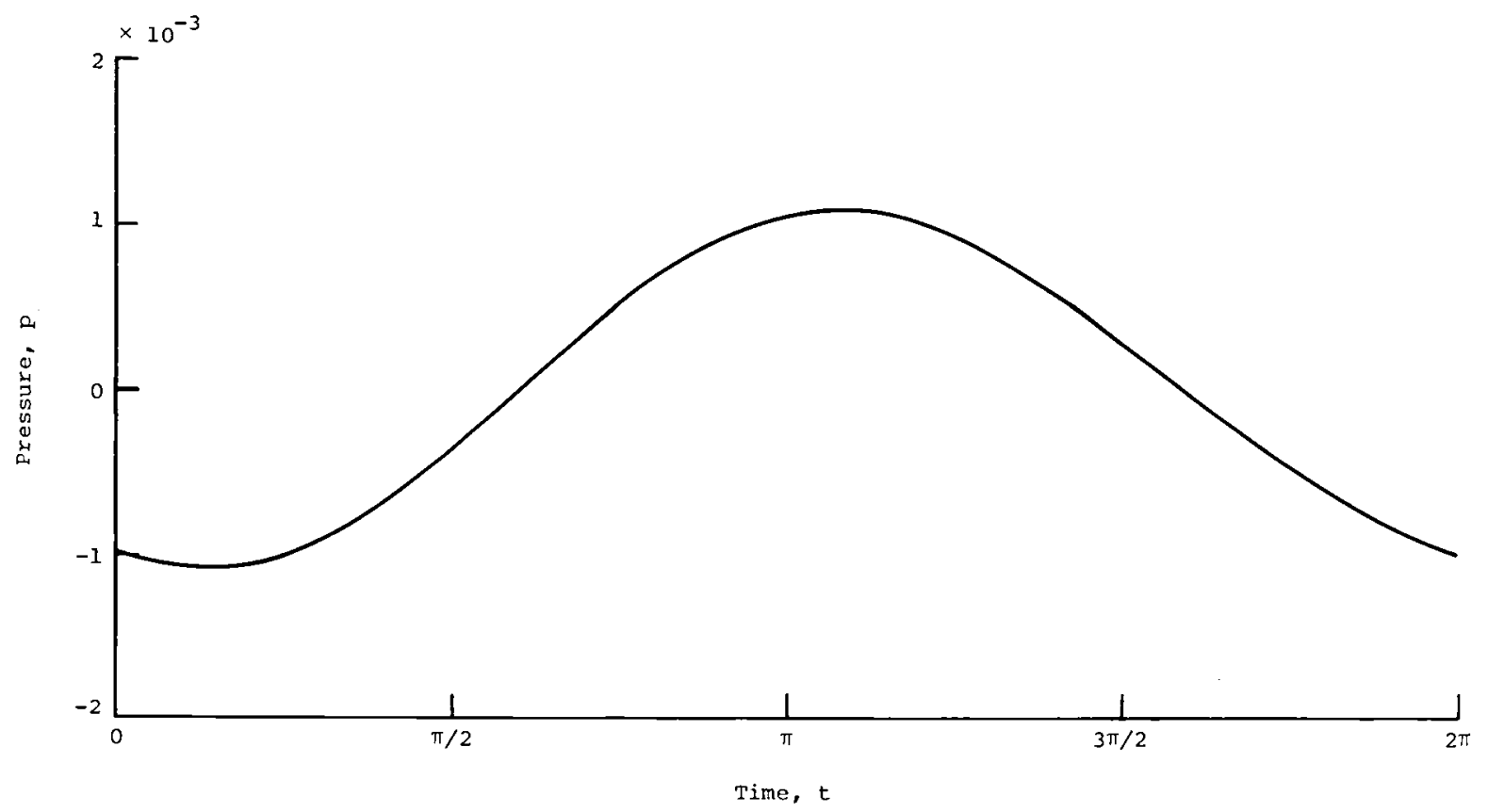

(b) Beginning of area variation; $x / L=0.25$.

Figure 6.- Acoustic pressure. 135-dB source; $M_{t}=-0.85 ; f=500 \mathrm{~Hz}$. 


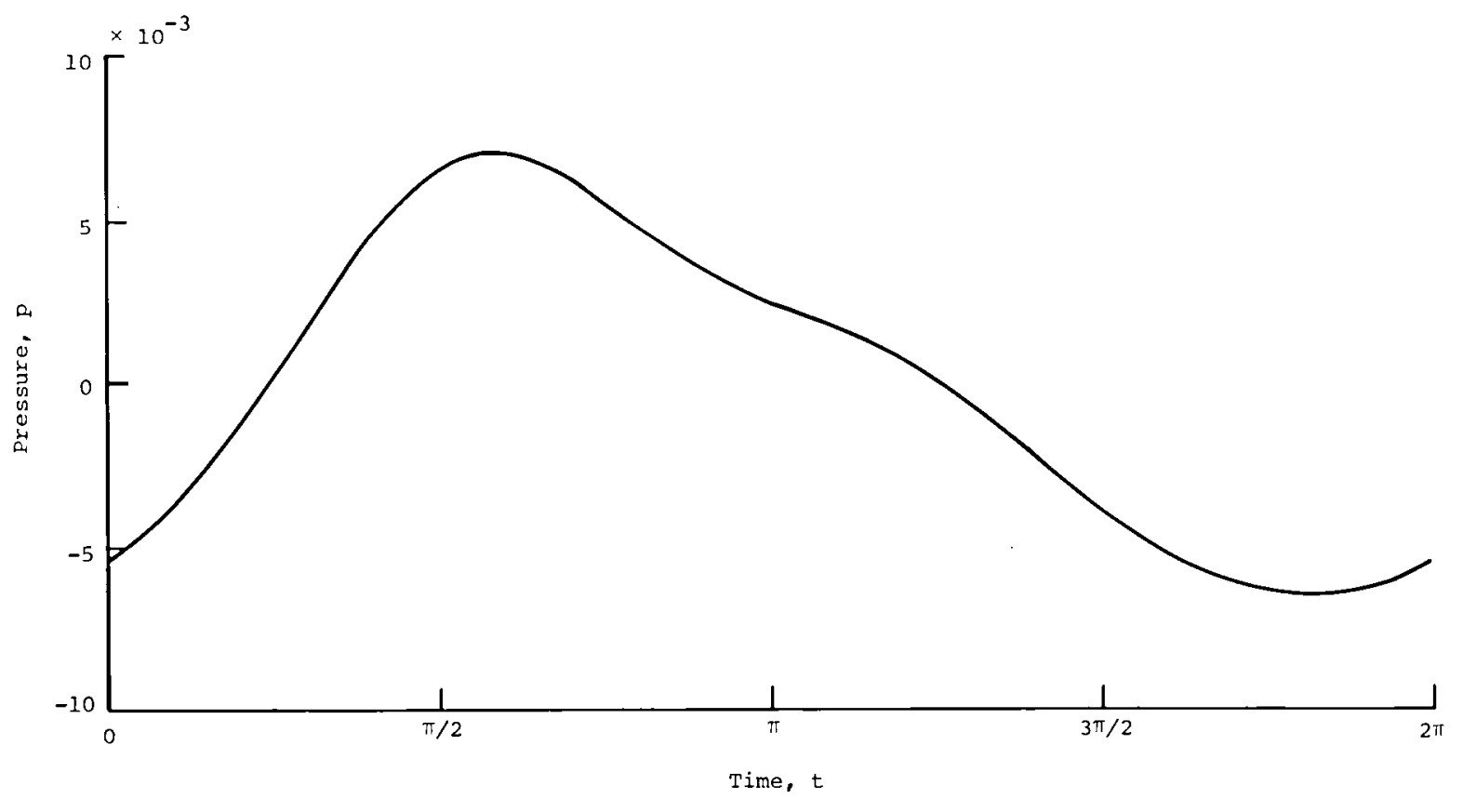

(c) Throat; $x / L=0.50$.

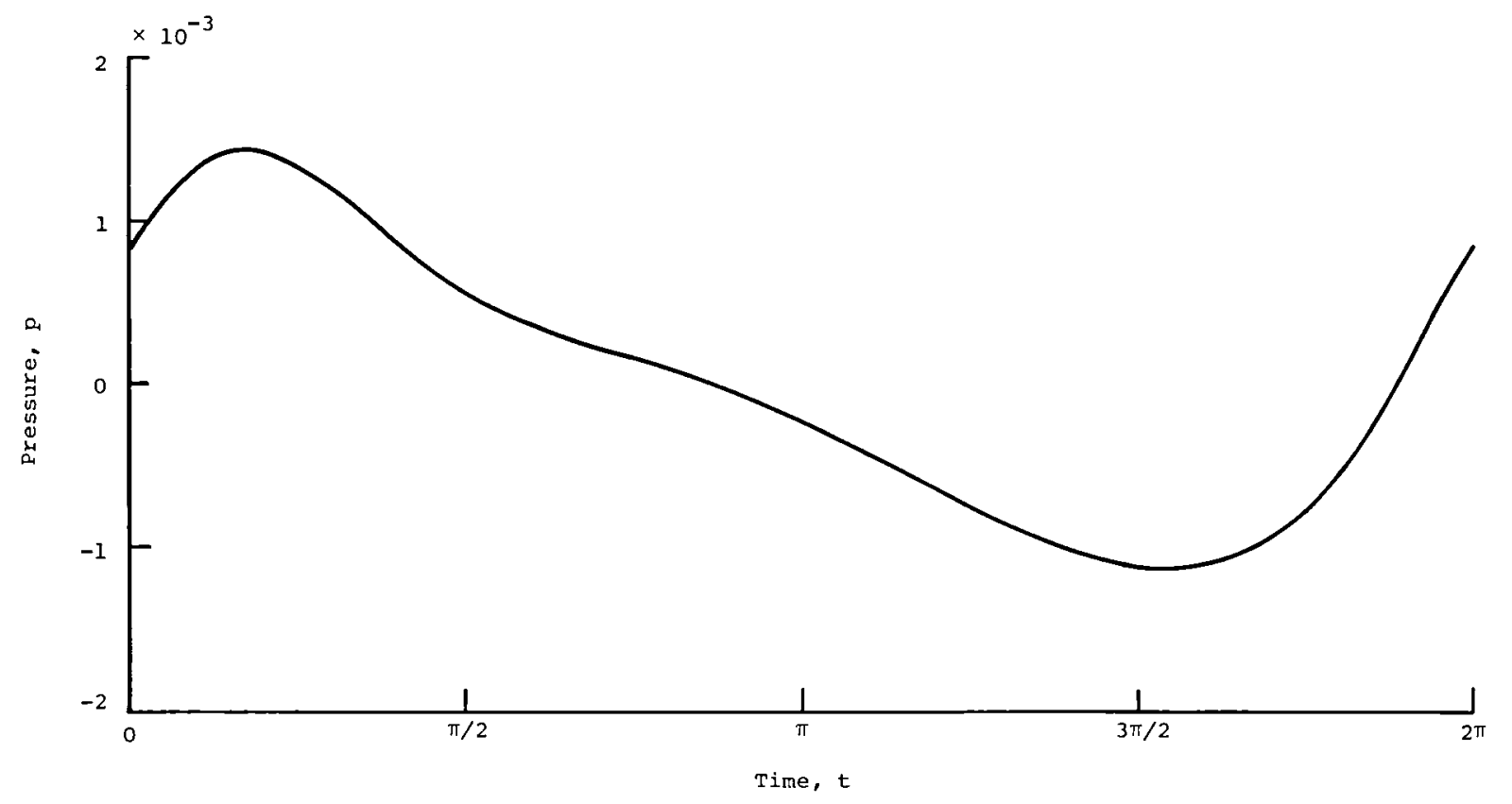

(d) End of area variation; $x / L=0.75$.

Figure 6.- Continued. 


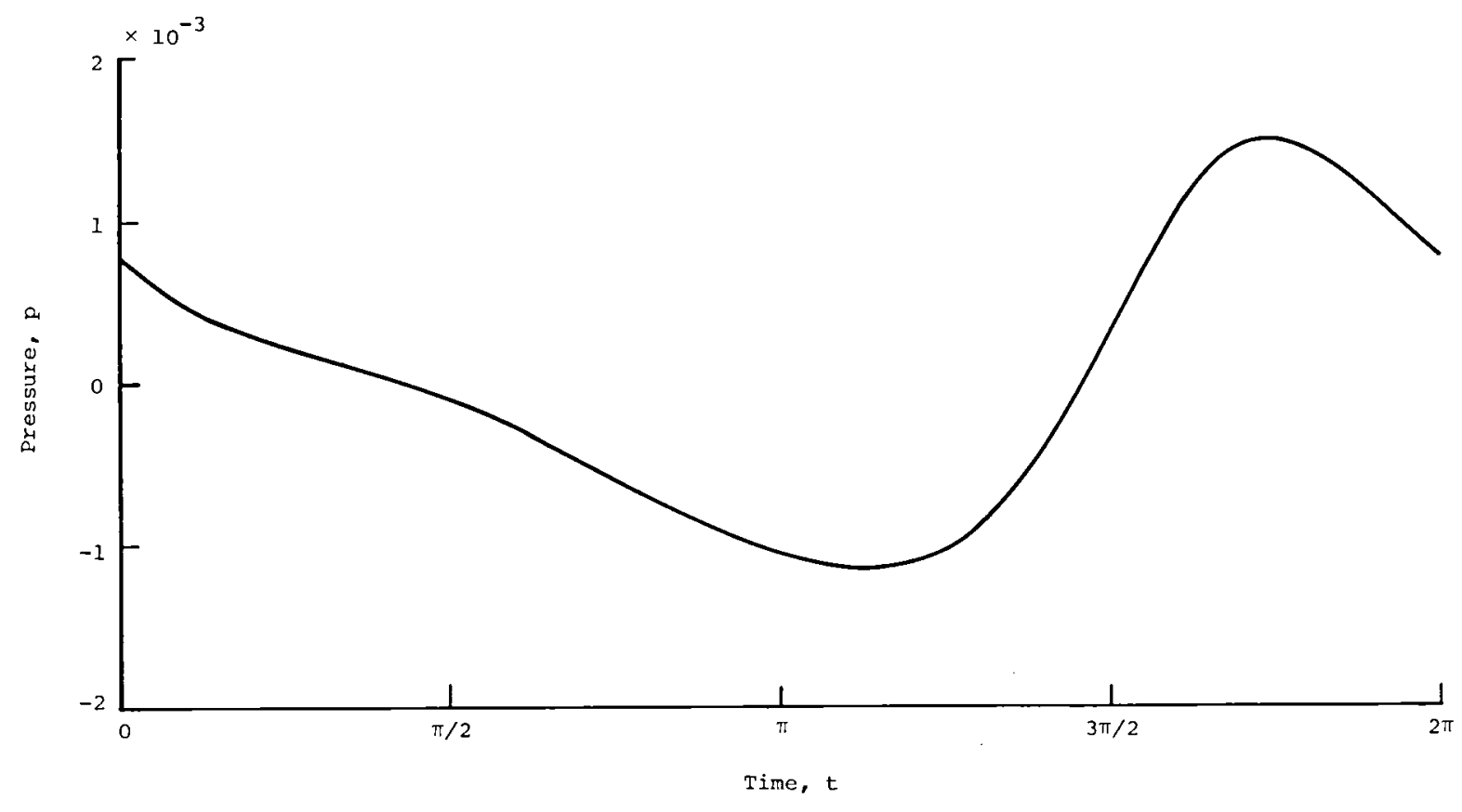

(e) Termination; $x / L=1.00$.

Figure 6.- Concluded. 


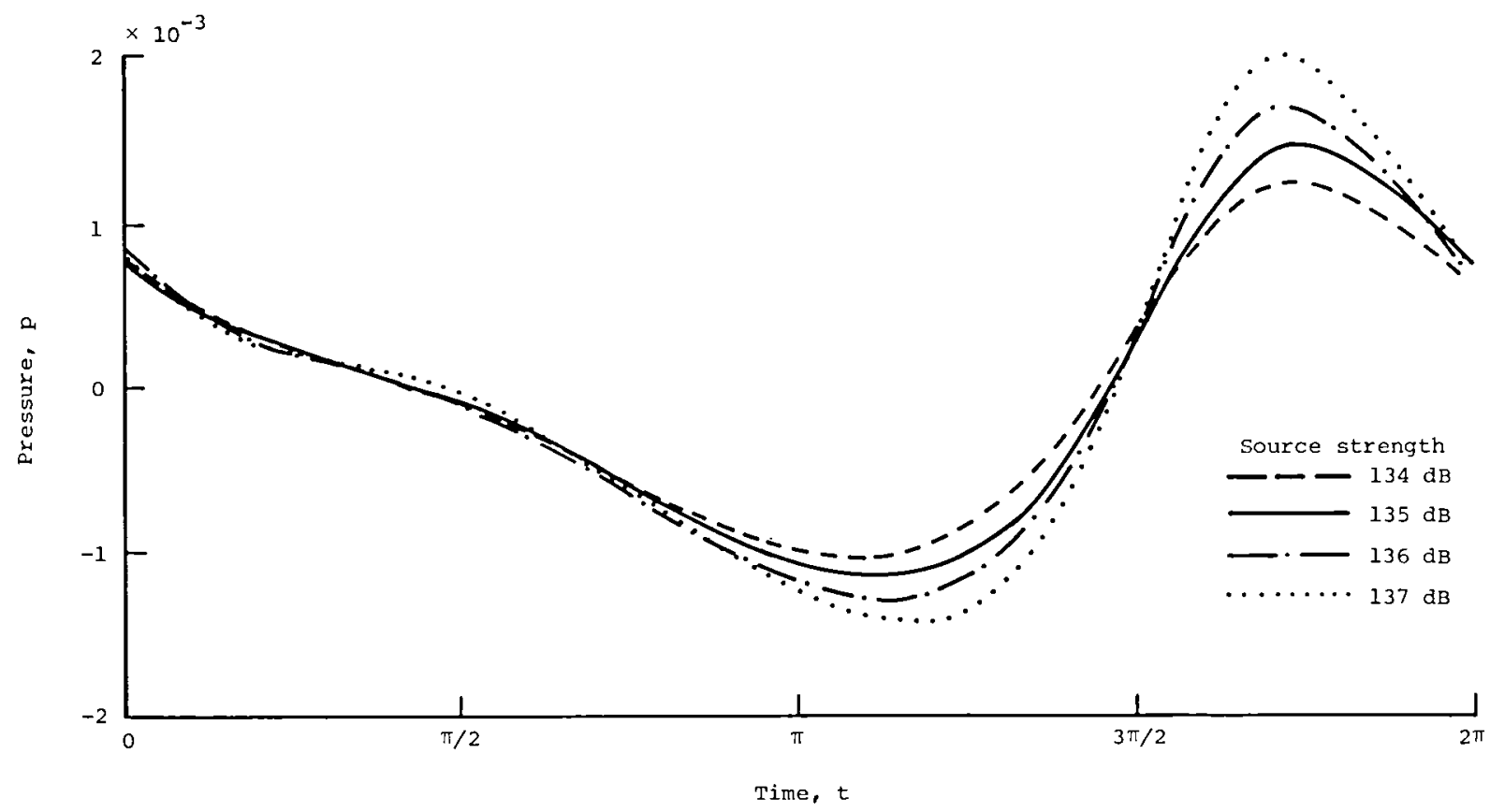
Figure 7.- Acoustic pressure at $x / L=1.00$ (termination). $M_{t}=-0.85 ;$
$f=500 \mathrm{~Hz}$.

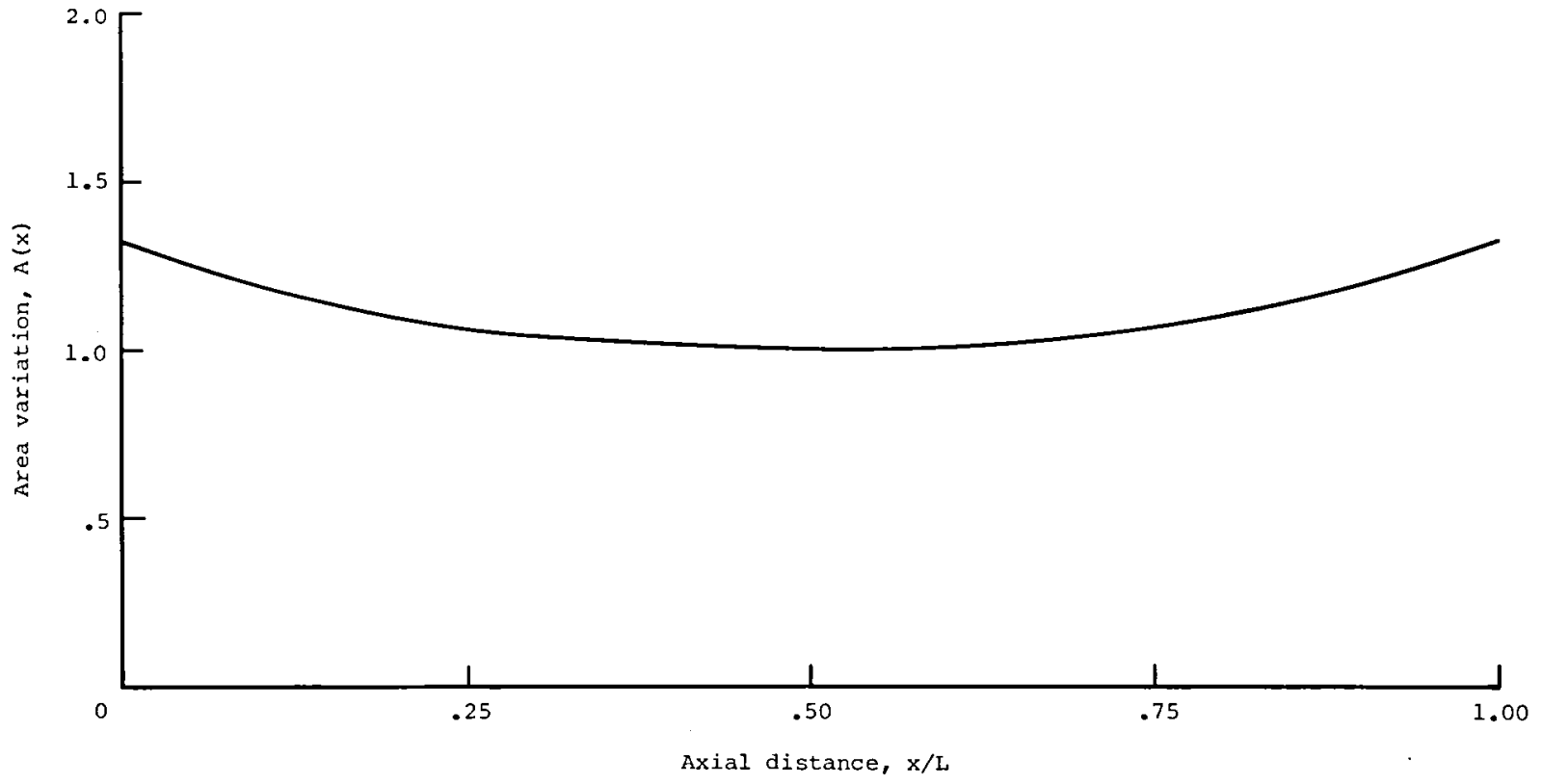

Figure 8.- Area variation used for comparison with asymptotic theory. 


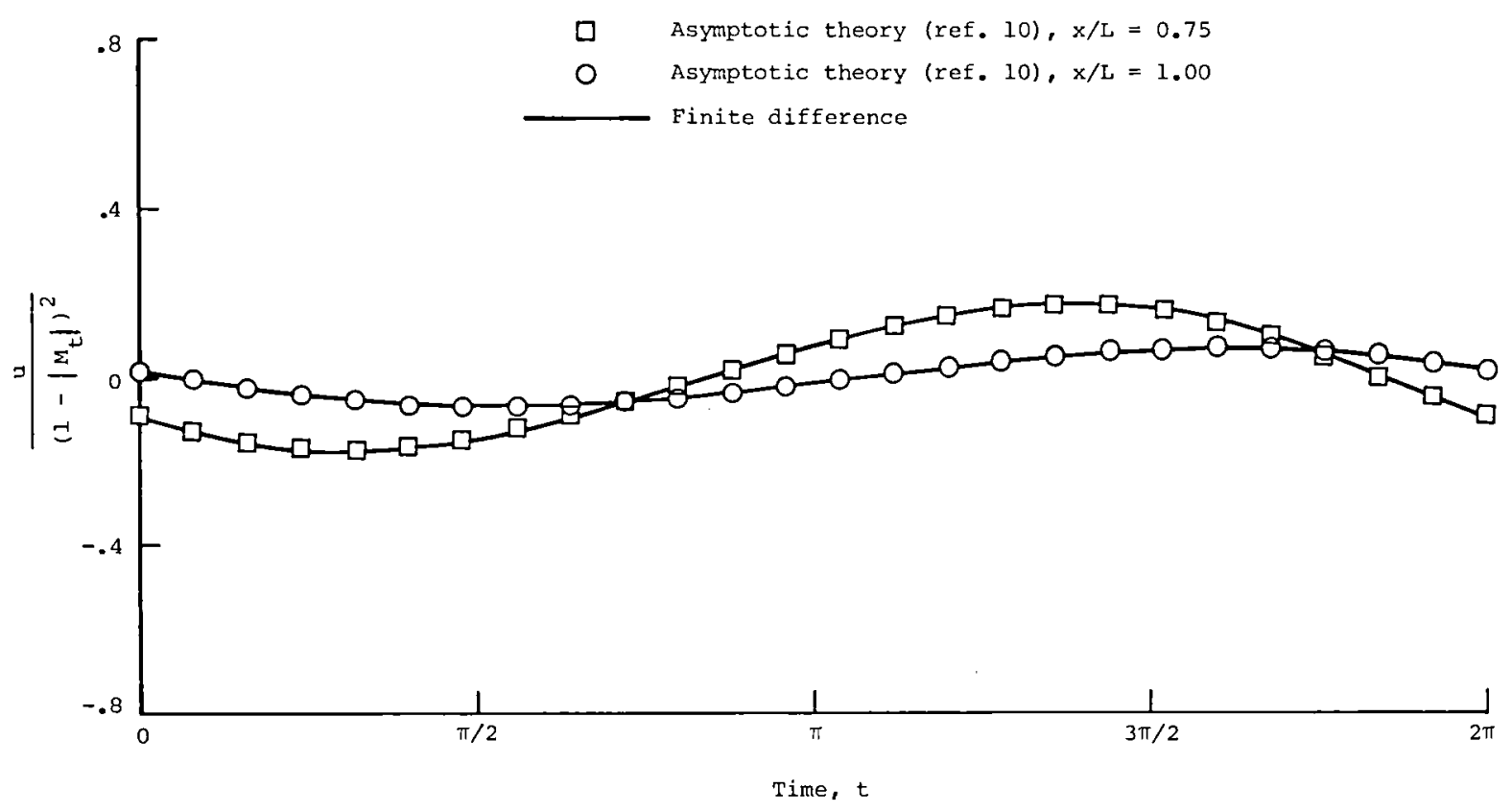

Figure 9.- Acoustic velocity. 130-dB source; $M_{t}=-0.90 ; f=452.2 \mathrm{~Hz}$.

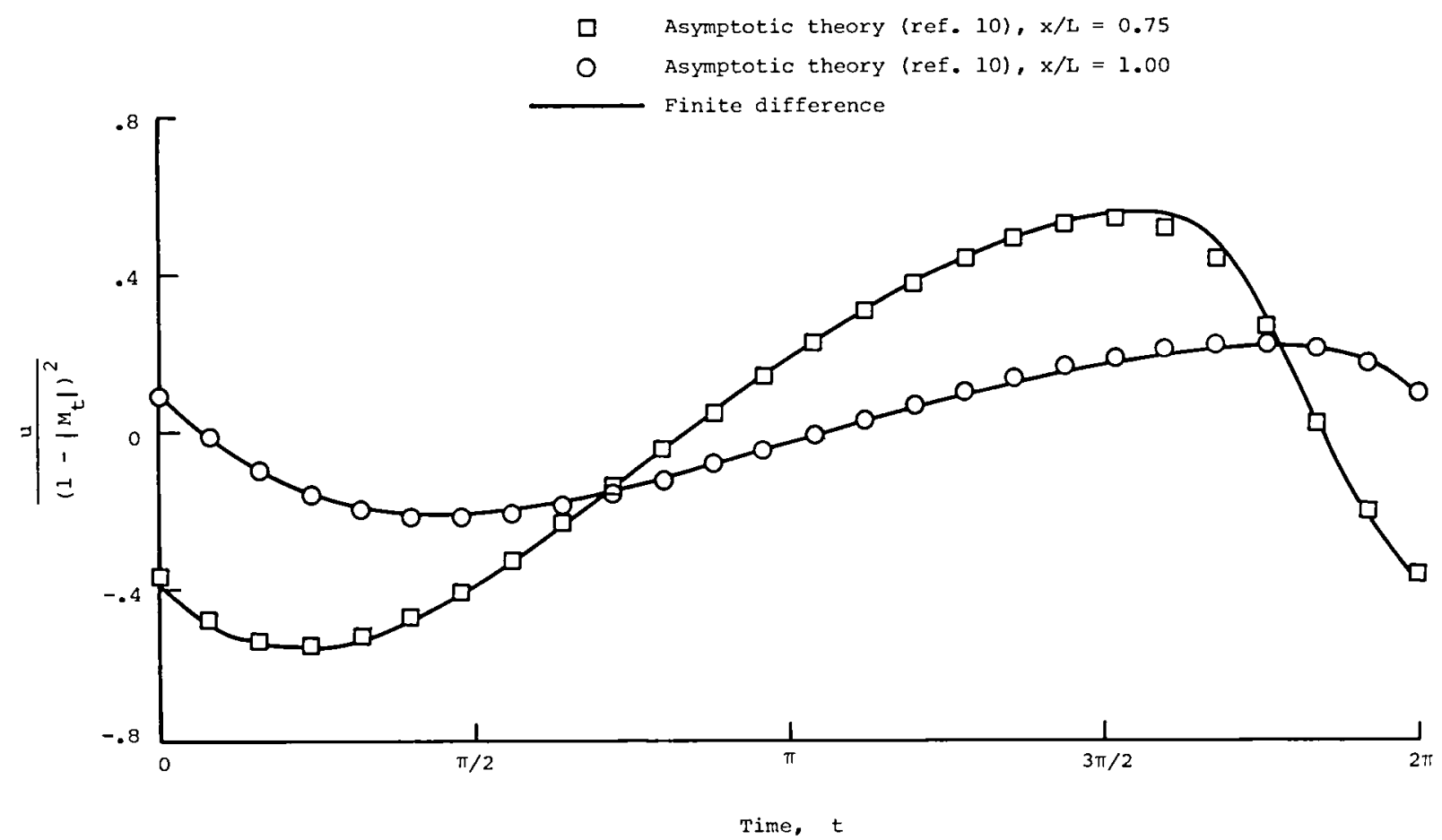

Figure 10.- Acoustic velocity. 140-dB source; $M_{t}=-0.90 ; f=452.2 \mathrm{~Hz}$. 


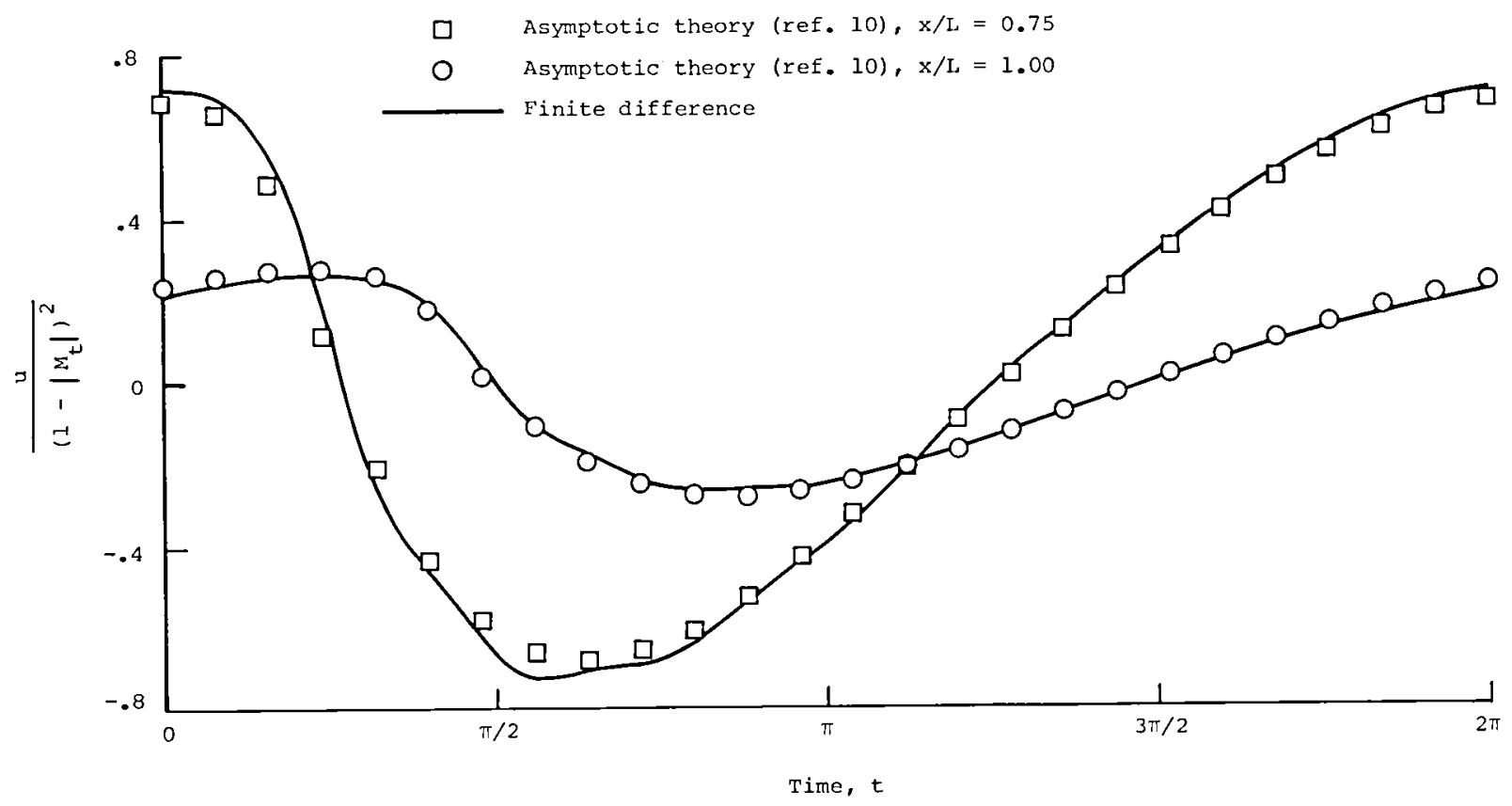

Figure 11.- Acoustic velocity. 130-dB source; $M_{t}=-0.95 ; \quad f=452.2 \mathrm{~Hz}$. 





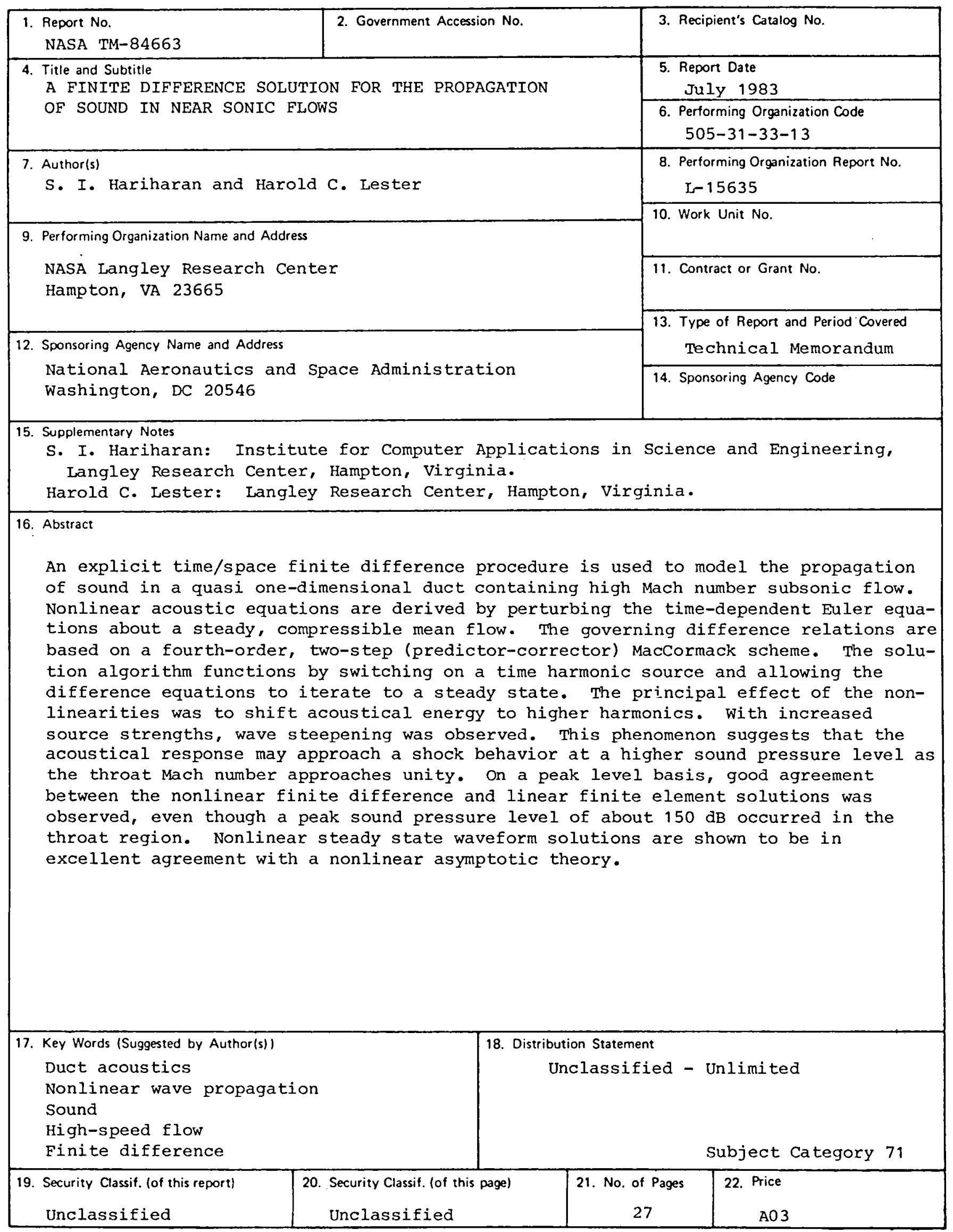


National Aeronautics and Space Administration

Washington, D.C.

20546

Official Business

Penalty for Private Use, $\$ 300$
Postage and Fees Paid

National Aeronautics and

Space Administration

NASA-451

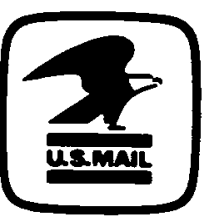

